\title{
Nonuniform Thermal Reversal in Single-Domain Patterned Media
}

\author{
Rok Dittrich, Member, IEEE, Thomas Schrefl, Member, IEEE, Dieter Suess, Werner Scholz, Member, IEEE, and \\ Josef Fidler, Member, IEEE
}

\begin{abstract}
Patterned media shows great potential for future ultrahigh-density magnetic recording. The thermal stability is determined by the relevant transition states. We apply the nudged elastic band method to calculate energy barriers and thermal reversal modes as a function of the island size and intergrain exchange coupling. Nonuniform reversal modes and transition states are found even in single-domain islands. The resulting energy barriers are significantly lower than those estimated from simplified reversal modes.
\end{abstract}

Index Terms-Energy barriers, intergrain coupling, minimum energy path, nonuniform reversal modes, nudged elastic band method, patterned media, single-domain particle, thermal stability.

\section{INTRODUCTION}

$\mathbf{T}$ HE currently used thin film magnetic recording media consist of small, single-domain magnetic grains, which are exchange isolated from each other. For an acceptable media signal-to-noise ratio, each data bit must contain a minimum number of grains. Grain diameters in the range of 7 to $20 \mathrm{~nm}$ and several tens to hundreds of grains in each written bit are currently used. To increase the storage density further, the multigrain bits and therefore each grain in each bit must be scaled down to smaller sizes. However, if the grain size becomes too small, the magnetization state cannot be retained against thermal decay due to the small energy barrier height. The magnetization will switch easily, leading to loss of recorded data.

Patterned media [1] have long been one of the most promising approaches toward breaking through the boundary of conventional continuous thin film media in magnetic information storage. In patterned media the bit structure is already predefined in the production of the disk. It then consists of discrete exchange isolated elements (e.g., array of thin squares separated by a nonmagnetic material). Ideally, the storage density is then equal to the surface density of the elements. Inside each element the polycrystalline grains are strongly exchange-coupled, and thus behaving like a large single magnetic grain. The single-domain magnetic element can be made of polycrystalline materials as well as single crystal or amorphous materials. Because the superparamagnetic limit applies to the whole island, and not to each of the many grains as in a conventional continuous multigrain bits, the

Manuscript received October 16, 2003.

The authors are with the Institute of Solid State Physics, Vienna University of Technology, 1040 Wien, Austria (e-mail: rok.dittrich@tuwien.ac.at).

Digital Object Identifier 10.1109/TMAG.2004.834204 volume and switching energy for the single-element bit in the patterned media are much larger than that of a single grain in conventional continuous media, allowing significant reduction in bit size. The minimum volume of the discrete element bit is still determined by the superparamagnetic limit, and could be as small as a few $\mathrm{nm}$ in diameter, depending on the magnetic properties of the material. A periodicity of $25 \mathrm{~nm}$ give an areal density of $1 \mathrm{~Tb} / \mathrm{in}^{2}$.

In this work, we apply the nudged elastic band (NEB) method to calculate energy barriers in patterned recording media. This method finds minimum energy paths and the corresponding energy barriers in high-dimensional energy landscapes and is shortly described in Section II.

\section{METHOD AND THEORY}

\section{A. Thermal Stability}

If the thermal energy $k_{B} T$ is comparable to the energy barrier $E_{B}$ that separates two local energy minima, direct simulations of the escape over the energy barrier using Langevin dynamics are possible. Unfortunately, this is no longer the case when $k_{B} T \ll E_{B}$ (either high energy barriers or low temperature limit) since the time to escape from the energy minimum grows exponentially with the barrier height. Here, the NEB method [2] turned out to be a powerful tool to directly compute the separating saddle points between stable states of the magnetization. The saddle points give the height of the energy barrier(s) which determines the switching probability between the stable states.

\section{B. NEB Method}

Henkelman and Jónsson proposed the NEB method to calculate minimum energy paths and energy barriers [2]. To calculate the minimum energy path between two local energy minima, an initial path is assumed (simple guess) between the states. The initial path is represented by a discrete sequence of images $M(k)$ and connects the initial magnetization state $M(i)=$ $\mathrm{M}(1)$ with the final magnetization state $M(f)=M(m)$. The index $k$ runs from 1 to $m$. The path is optimal, if for any image $M(k)$ the gradient of the energy only points along the path. In other words: the component of the energy gradient normal to the path is zero. This path is called minimum energy path, which means that the energy is stationary for any degree of freedom perpendicular to the path (see solid line in Fig. 1). The minimum energy path typically represents the path with the greatest statistical weight. From this path, statistical quantities as for example transition rates for the thermally induced magnetization reversal 


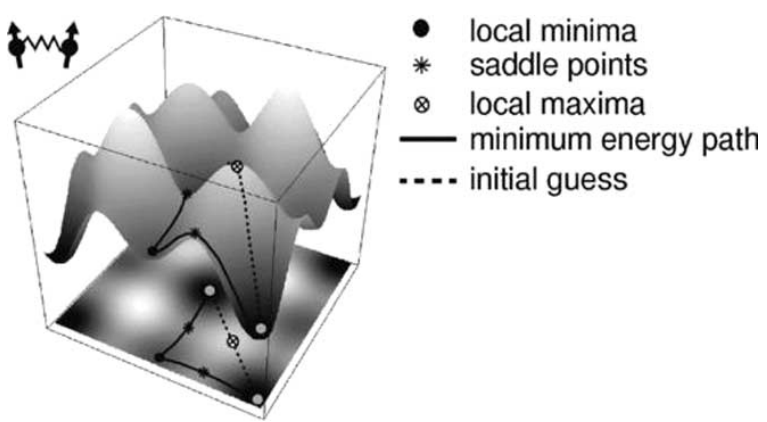

Fig. 1. Two-dimensional energy landscape representing two anisotropic magnetic moments with weak exchange coupling between them. The dashed line shows the initial guess, and the solid line, the resulting minimum energy path obtained from the NEB method.

can be estimated. We use finite-element micromagnetics [4] to model the structures and to calculate energies, energy gradients, and effective magnetic fields. The minimum energy path is then found using an iterative scheme. In each step, the images move toward lower energy in a direction perpendicular to the path. This iterative scheme is numerically very ineffective. Instead, we solve a system of ordinary differential equations using the implicit ordinary differential equation solver CVODE [5]. Details on the implementation of the NEB method can be found in [2] and [3].

\section{RESULTS}

\section{A. Intergranular Coupling Strength}

In the first step, we calculated the energy barrier as a function of the intergrain exchange coupling strength for a $23 \mathrm{~nm}$ large and $10 \mathrm{~nm}$ thick island. Such small islands are required to approach an areal density of $1 \mathrm{~Tb} / \mathrm{in}^{2}$ [6]. The grain boundary thickness was $0.7 \mathrm{~nm}$, the grain size $7 \mathrm{~nm}$, magnetocrystalline anisotropy constant $K_{u}=200 \mathrm{~kJ} / \mathrm{m}^{3}$, and exchange constant $A_{\text {bulk }}=10 \mathrm{pJ} / \mathrm{m}$. The used model is shown in Fig. 3 on the left. The patterned element consists of 16 grains. In the micromagnetic model, the grains are subdiscretized into finite elements. The easy axes directions of the grains are normal to the plane with a dispersion of the angles of $8^{\circ}$ around the plane normal.

Fig. 2 compares the minimum energy paths for weak, intermediate, and strong intergranular exchange coupling strength. At a weak intergrain exchange coupling $\left(J=0.3 \mathrm{~mJ} / \mathrm{m}^{2}\right)$, the individual grains in the island all switch individually. The thermal stability is mainly determined by the thermal stability of the individual grains. At an intermediate coupling strength, $J=1 \mathrm{~mJ} / \mathrm{m}^{2}$, the grains start to behave like one large grain and not more as an ensemble of individual grains. The resulting energy barrier is much higher than for weak coupling. The reversal starts with nucleation on one corner and the expansion of the reversed domain. Note that a metastable state is present where the magnetization is in a two-domain state. At even stronger coupling $\left(J=5 \mathrm{~mJ} / \mathrm{m}^{2}\right)$, the metastable two-domain state disappears (see Fig. 2). Although the energy minima are the uniform single-domain state, the transition state (saddle point) is a nonuniform two-domain state ("S1" in Fig. 2). If we would

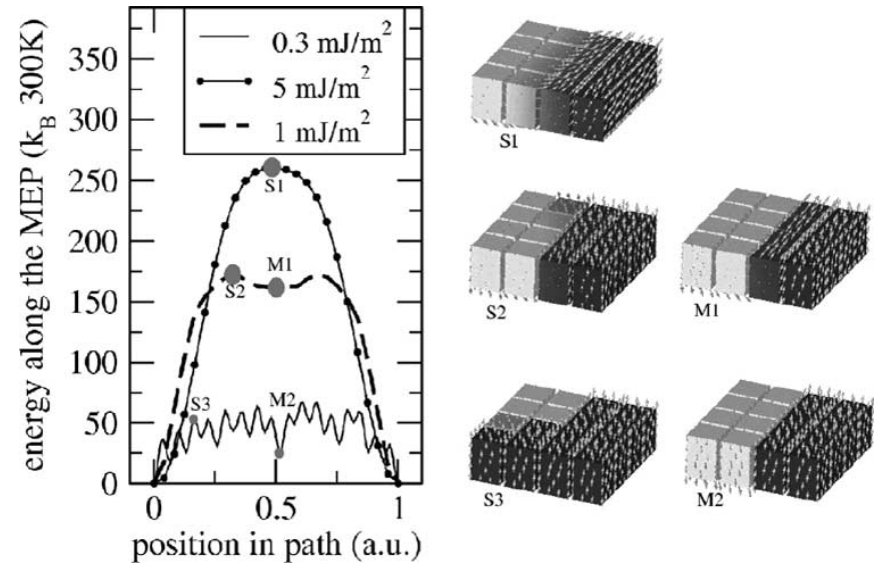

Fig. 2. Comparison of minimum energy paths (MEPs) for weak $\left(J=0.3 \mathrm{~mJ} / \mathrm{m}^{2}\right)$, intermediate $\left(J=1 \mathrm{~mJ} / \mathrm{m}^{2}\right)$, and strong $\left(J=5 \mathrm{~mJ} / \mathrm{m}^{2}\right)$ intergrain exchange coupling strength. The most important magnetization states (saddle points and energy minima) are shown on the right. The color code corresponds to the out-of-plane magnetization (dark...up, bright. . down). The element is fully magnetized up at the positions " 0 " in the MEPs and fully magnetized down at position "1." These are the absolute energy minima which are used to store one bit of data.

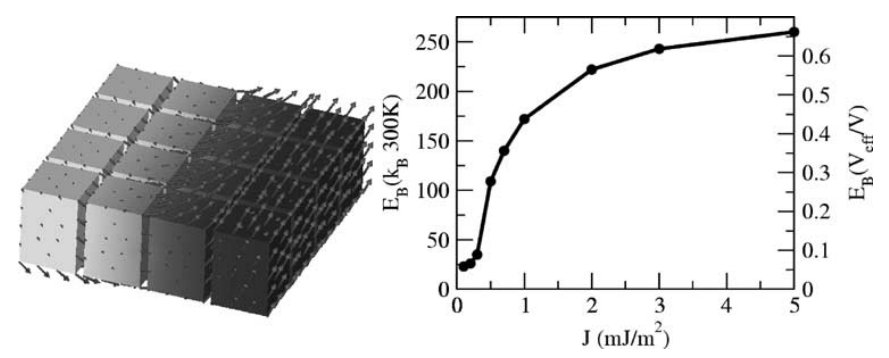

Fig. 3. (Left) Model of one discrete patterned element which consists of 16 exchange coupled subgrains. Shown is the nonuniform transition state (saddle point) for the zero field thermal reversal at a strong coupling strength ( $J=$ $5 \mathrm{~mJ} / \mathrm{m}^{2}$ ). (Right) The curve shows results obtained with the NEB method. The energy barrier of one patterned element is plotted versus the intergrain exchange coupling strength $J$.

simply assume uniform rotation of the magnetization, the energy barrier would write $E_{B}=K_{u} V$. The real transition state is however still nonuniform as shown, and the resulting energy barrier is thus only $66 \% K_{u} V$. Fig. 3 summarizes the results giving the energy barrier as a function of the strength of the intergrain exchange coupling.

\section{B. Dependence of the Energy Barrier on the Island Size}

In the next step, we calculate the dependence of the energy barrier on the size of the islands. We assume squared patterned elements with perfect exchange coupling of the grains within the element. The same material parameters as before were used $\left(K_{u}=200 \mathrm{~kJ} / \mathrm{m}^{3}, A=10 \mathrm{pJ} / \mathrm{m}\right)$.

In the following, we assume perfectly coupled grains. The energy barrier was calculated as a function of the island size and the results were compared with a simple analytical estimate. Fig. 2 shows that the thermal reversal of the patterned island basically involves the creation of a two domains. Due to the high magnetocrystalline anisotropy, the height of the energy barrier is mainly determined by the domain wall energy of the 
two-domain state. In the following analytical estimation, we neglect the contribution from the magnetostatic interaction. In this simple model, the energy barrier height is equal to the domain wall energy

$$
E_{1}=\operatorname{Area} \cdot 4 \sqrt{A \cdot K_{u}} \text {. }
$$

Area is the total area of the domain wall. Below a critical size, this energy will be higher than the energy barrier for coherent rotation which is $E_{2}=$ Volume $\cdot K_{u}$, where Volume is the total volume of the island. For an island with lateral extension $L$ and thickness $t$, we have Area $=L \cdot t$ and Volume $=$ $L \cdot L \cdot t$. If we set $E_{1}=E_{2}$, we obtain the critical size for coherent rotation

$$
L_{\text {critical }}=4 \sqrt{\frac{A}{K_{u}}}=4 \delta_{0}
$$

Note that the result is independent of the height of the island, and is equal to four times the Bloch parameter $\delta_{0}$. If the island size is smaller than $4 \delta_{0}$ we expect uniform rotation. With $A=$ $10 \mathrm{pJ} / \mathrm{m}, K_{u}=200 \mathrm{~kJ} / \mathrm{m}^{3}$, and $t=10 \mathrm{~nm}$, the critical island size $L_{\text {critical }}$ becomes $28.2 \mathrm{~nm}$.

The numerical calculation of the minimum energy paths and energy barriers using the NEB method shows that there is a continuous transformation between the inhomogeneous reversal and the uniform rotation mode as the size decreases. Nonuniform reversal modes are found even for an island size of $20 \mathrm{~nm}$, which is well below the critical size $L_{\text {critical }}$ (2). Fig. 4 shows the size dependency of the energy barrier. The energy barrier is given in units of $K_{u} V$. This is equal to an effective switching volume, $V_{\text {eff }}$, divided by the total volume $\left(E_{B} / K_{u} V=V_{\text {eff }} / V\right)$. For all island sizes, the analytical model overestimates the energy barrier height. The strongest deviation is found when the island size is near the calculated critical size. At $20 \mathrm{~nm}$, island size the calculated energy barrier is $17 \%$ smaller than in the uniform rotation model (Fig. 4). Even at this size, the reversal mode is still slightly nonuniform.

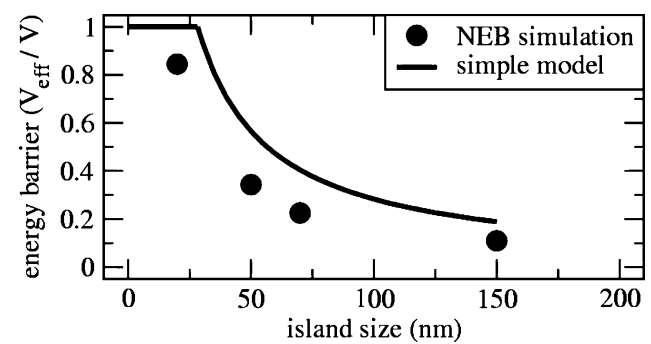

Fig. 4. Energy barrier in units of $K_{u} V$ as a function of the island size for a squared island. The analytical model always overestimates the energy barrier as compared to the micromagnetic energy barrier computation. Moreover, it can be seen that the reversal mode is inhomogeneous even in the regime $(<25 \mathrm{~nm})$ where uniform rotation is expected. The height of the islands was $10 \mathrm{~nm}, K_{u}=$ $200 \mathrm{~kJ} / \mathrm{m}^{3}, A=10 \mathrm{pJ} / \mathrm{m}$.

\section{CONCLUSION}

Energy barriers were calculated in patterned elements as a function of the size and the intergrain exchange coupling strength. The full micromagnetic approach is a rigorous way of computing energy barriers. Nonuniform transition states are found even on length scales where the magnetization can only occupy a single-domain state. The resulting energy barriers are thus significantly lower than as extracted from the simple uniform rotation model.

\section{REFERENCES}

[1] R. L. White, R. M. H. New, and R. F. W. Pease, "Patterned media: a viable route to $50 \mathrm{Gbit} / \mathrm{in}^{2}$ and up for magnetic recording?," IEEE Trans. Magn., vol. 33, p. 990, Jan. 1997.

[2] G. Henkelman, B. P. Uberuaga, and H. Jónsson, "Improved tangent estimate in the NEB method for finding minimum energy paths," J. Chem. Phys., vol. 113, p. 9901, 2000.

[3] R. Dittrich, T. Schrefl, D. Suess, W. Scholz, H. Forster, and J. Fidler, "A path method for finding energy barriers and minimum energy paths in complex micromagnetic systems," J. Magn. Magn. Mater, vol. 250, p. 12, 2002.

[4] D. Suess, V. Tsiantos, T. Schrefl, J. Fidler, W. Scholz, H. Forster, R. Dittrich, and J. Miles, "Time resolved micromagnetics using a preconditioned time integration method," J. Magn. Magn. Mater, vol. 248, p. 298, 2002.

[5] S. D. Cohen and A. C. Hindmarsh, "CVODE, a stiff/nonstiff ODE solver in C," Comput. Phys., vol. 10, p. 138, 1996.

[6] A. Moser, K. Takano, D. T. Margulies, M. Albrecht, Y. Sonobe, Y. Ikeda, S. H. Sun, and E. E. Fullerton, "Magnetic recording: advancing into the future," J. Appl. Phys. D, vol. 35, no. 19, pp. R157-R167, 2002. 